\title{
IL-17 in primary knee osteoarthritis and its relation with severity of the disease
}

Objective: Is to measure serum level of interleukin 17 and correlate its level with disease severity in patients with primary knee OA.

Methods: Serum was collected from patients with primary knee osteoarthritis and age- and sex-matched healthy control subjects. IL-17 was quantified by enzyme-linked immunosorbent assay. Osteoarthritis severity and grade were assessed using the Lequesne index and Kellgren and Lawrence (KL) grading system, respectively.

Results: Serum IL-17 concentrations are significantly higher in patients $(1039.5 \mathrm{pg} / \mathrm{ml})$ than in controls $(55 \mathrm{pg} / \mathrm{ml})$ with $\mathrm{p}$ value $<0.001$. This is significantly positively correlated with degree of WOMAC, pain scale, Lequesne index, $\mathrm{KL}$ grade and with MRI grades.

Conclusions: According to the results of the present study we can conclude that there is a strong relation between serum level of IL-17 and primary knee osteoarthritis and this may reflect the degree of severity of the disease to some extent

Keywords: osteoarthritis • interleukin • cytokines

\section{Introduction}

Osteoarthritis (OA) is the commonest joint disease leading to pain and disability to its affected patients. It is not only one disorder; rather it is a group of diseases with many different hidden pathological pathways. It is a disorder that increases as time passes by. Even though cartilage destruction is a main character of OA, it's also characterized by narrowing of joint space, marginal osteophytes formation, subchondral bone remodelling and synovial inflammation. All of these characteristics are considered strongly responsible for the disease progression [1].

The most common findings in the history and physical examination of the patients with knee $\mathrm{OA}$ are mechanical knee pain, gelling knee pain, crepitus on knee motion, bony tenderness and bony enlargement in the joint line [2].

Despite its popularity, the origin of OA disease remains unclear till now. Among the studies that discussed the initiation and continuation of OA, two aspects appear to be of great importance, the absence of a balanced cytokines system, including proinflammatory (i.e. IL-17, IL-1 and TNF) and anti-inflammatory (i.e. IL-10, IL-4, IL-1 receptor (IL1R) antagonist) soluble factors at the cartilage tissue, and the alteration in the appearance of chemokines receptors in chondrocytes [1].
IL-17 is the original member of a new group of cytokines (IL-17 A-F); it has strong proinflammatory characteristics since it shows similar characteristics to those of TNF-a and IL-1. IL-17 most common sources come from $\mathrm{CD}+4$ effector $\mathrm{T}$ cells and Th-17 lineage, however many other sources have been recognized, such as $\mathrm{CD}+8 \mathrm{~T}$ cells, eosinophils, and neutrophils [3].

Additionally, IL-17 has proven to disturb the extracellular matrix (ECM) equilibrium either alone or by the help of other pro-inflammatory cytokines or adipocytokines [3]. Furthermore, IL-17 can lead to the survival of synovium fibroblasts and inflammation cells and also enhances the expression of the monocytes chemo attractants C - C motif chemokines Ligand 7 (CCL7) and CCL2 [4]. In addition, it has the ability to stimulate chemokines production by osteoarthritic chondrocytes this can be suppressed by the addition to anti-IL-17 monoclonal antibody [5].

All of the above evidence indicates that IL17 is capable of encouraging the secretion of many other cytokines, and may participate in the activation of multiple catabolic pathways resulting in cartilage and tissue damage in osteoarthritis [6]. The aims of this study was to measure serum level of interleukin 17 inpatients with primary knee $\mathrm{OA}$ and correlate its level to disease severity.

\section{Sahar A Mohamed', Nanis 0 Neseem $^{* 1}$, Shereen S Metwally ${ }^{2}$ \& Seif El Dein M Farag ${ }^{1}$ \\ 'Physical medicine, Rehabilitation and Rheumatology Department, Faculty of medicine, Mansoura University, Egypt ${ }^{2}$ Clinical Pathology Department, Faculty of medicine, Mansoura University, Egypt \\ *Author for correspondence: nanisonsy281@gmail.com}




\section{Subject and methods}

This study was conducted on 40 patients with primary knee osteoarthritis attending the inpatient and outpatient clinics of Physical Medicine, Rehabilitation and Rheumatology Department, Mansoura University. All patients were selected according to EULAR evidences based criteria for knee OA diagnosis [7]. In addition to 47 apparently healthy age and sex matched subjects considered as control group. Written consent was collected from every person participating in this study.

\section{Exclusion criteria}

Include Patients having systemic autoimmune disease, secondary osteoarthritis, chronic liver and kidney disease.

\section{Clinical examination}

All patients were subjected to full history and clinical examination in addition to assessment of presence of crepitus, tenderness on a rating scale 0-3, according to Gunn [8], Swelling: Localized or diffuse, and bony prominence, Quadriceps wasting - Evaluation, assessment of joint effusion - graded on a scale of $0-3$ [9], range of motion, special tests to assess ligaments and menisci [10], pain assessment using VAS pain scale [11], assessment of disease function using Western Ontario and McMaster (WOMAC) OA index [12], assessment of disease severity using Lequesne scale [13], X-ray results were calculated and scored according to the Kellgren and Lawrence (KL) radiological scale [14], and Magnetic Resonance Imaging was graded as a measure of cartilage degradation using WORMS score for articular cartilage [15]:

Grade 0: Normal.

Grade 1: Focal cartilage defects without overall thinning.

Grade 2: Incomplete loss of the cartilage thickness (less than $50 \%$ of the area).

Grade 3: Multiple regions of total loss of thickness, from $50 \%-75 \%$ of the area.

Grade 4: Global total loss of cartilage thickness (more than $75 \%$ of the region).

Investigations

- Radiological examination including X-ray and Magnetic Resonance Imaging.

- Laboratory investigations including: Complete blood count, Erythrocyte
Sedimentation Rate [ESR] [16], C-Reactive Protein (CRP), and serum Interleukin-17 level.

\section{Assessment of serum IL-17 level}

Whole blood $(5 \mathrm{ml})$ was collected in vacutainer tubes, allowed to clot for $30 \mathrm{~min}$. at room temperature and then centrifuged for $15 \mathrm{~min}$ at $1000 \mathrm{~g}$. The resulting serum was removed and stored at $-80^{\circ} \mathrm{C}$ until use. Serum level of interleukin-17 was measured using Sandwich ELISA technique, according to the instruction of the manufacturer (NOVA, Beijing China).

\section{Principle}

Standards and samples were added to the appropriate micro Elisa strip plate wells which are pre-coated with antibody specific to IL-17. Then a Horseradish peroxidase (HRP)-conjugated $\mathrm{Ab}$ specific for IL-17 was added to each well and incubated. Free components were washed away. The TMB substrate dissolvent was added to every well. Only those wells that contain IL-17 and HRP conjugated IL-17 antibody appeared blue in color and then turned yellow after adding the stop solution. The Optical Density (OD) was measured spectrophotometrically at a wavelength of $450 \mathrm{~nm}$. The OD value is proportional to the concentration of IL-17. The concentration of IL17 in the samples was calculated by comparing the optical density of the samples to the standard curve.

\section{Statistical analysis}

Data were evaluated statistically by the Statistical Package for Social Sciences (SPSS) version 20 (SPSS, 2011). Qualitative data were explained in percentages and numbers with Chi-Square test used for comparison. Quantitative data were represented as median and range for nonparametric data or mean \& standard deviation for parametric data after testing normality by Kolmogorov-Smirnov test. Mann-Whitney tests were used for comparison between groups for (non-parametric variables) and Student t-test (for parametric ones). Spearman correlation between non parametric continuous variables. "p value $\leq 0.05^{\text {" }}$ was considered to be statistically significant. All tests were 2-tailed.

\section{Results}

This study included 40 primary osteoarthritis patients, they were $6(15.0 \%)$ males and 34 $(85.0 \%)$ females with a mean age of $47.58 \pm 6.8$ and 47 matched healthy for gender and age [ 6 $(12.8 \%)$ males and $41(87.2 \%)$ females mean 
age of $44.1 \pm 5.9]$ respectively. Distribution of studied patients according to different indices: WOMAC 96 Median $=46.0$, Leqense scale disease severity Median $=10$. Pain scale Mean \pm $\mathrm{SD}=6.8 \pm 1.7$ (Table 1$)$.

Comparison of serum IL-17 level between patients and controls shows statistically significant higher IL-17 level in OA patients compared to controls, $\mathrm{P}<001$ (Table 2). Table 3 shows statistically significant strong positive correlation between interleukin-17 and WOMAC ( $\mathrm{Rs}=0.99, \mathrm{P}<0.001)$, Lequesne scale $(\mathrm{R} s=0.99, \mathrm{P}<0.001)$ and pain scale $(\mathrm{R} s=0.85$, $\mathrm{P}<0.001)$.

Table 4 showed distribution of studied cases according to Kelgrence and Lawrence grading. Figure 1 shows statistically significant strong positive correlation between the level of interleukin-17 and Kellgren and Lawrence scale used as a grade for X-ray ( $R s=0.93, \mathrm{p}<0.001)$. Figure 2 showed statistically significant strong positive correlation between serum level of interleukin-17 and MRI grades as a measure of cartilage degradation $(\mathrm{Rs}=0.73, \mathrm{p}<0.001)$.

\section{Discussion}

The results of this study showed that all OA patients had different degrees of tenderness as $57.5 \%$ of patients had tenderness and winced during examination and further $25 \%$ of them withdrew their legs during examination due to severe tenderness. However most of patients (85\%) did not have any grade of effusion during examination. In the present study, the comparison among the serum level of interleukin-17 in OA patients and control group revealed that they were higher in osteoarthritis patients $\mathrm{p}<0.001$. This is in agreement with $[17,18]$.

Table 1. Distribution of studied cases according to different indexes.

\begin{tabular}{|c|c|}
\hline & $\mathbf{n = 4 0}$ \\
\hline WOMAC 96 & 46 \\
Median (Min-Max) & $(13.0-73.0)$ \\
\hline $\begin{array}{c}\text { Leqense scale disease severity } \\
\text { Median (Min-Max) }\end{array}$ & 10 \\
\hline Pain scale & $(3.0-19.0)$ \\
\hline Mean \pm SD & $6.8+1.7$ \\
\hline
\end{tabular}

Table 2. Comparison of serum level IL-17 between patients and controls.

\begin{tabular}{|c|c|c|c|}
\hline & $\begin{array}{c}\text { Patients } \\
\mathbf{n = 4 0}\end{array}$ & $\begin{array}{c}\text { Controls } \\
\mathbf{n = 4 7}\end{array}$ & $\begin{array}{c}\text { Test of } \\
\text { significance }\end{array}$ \\
\hline $\begin{array}{c}\text { IL-17 } \\
\text { Median }\end{array}$ & $\begin{array}{c}1039.5 \\
(145.0-\end{array}$ & $\begin{array}{c}55 \\
(3.0-\end{array}$ & $\begin{array}{c}\mathrm{Z}=7.99 \\
\mathrm{p}<0.001\end{array}$ \\
$\mathrm{Pg} / \mathrm{ml}-\mathrm{max})$ & $2125.0)$ & $180.0)$ & \\
\hline
\end{tabular}

\begin{tabular}{|c|c|c|}
\hline & IL 17 & \\
\hline & Rs & $\mathbf{P}$ \\
\hline WOMAC & 0.99 & $<0.001$ \\
\hline Lequesne scale & 0.99 & $<0.001$ \\
\hline Pain scale & 0.85 & $<0.001$ \\
\hline
\end{tabular}

\begin{tabular}{|c|c|c|}
\hline Kelgrence and Lawrence grading & $\mathbf{N}$ & $\%$ \\
\hline${ }^{*} 0$ & 0 & 0 \\
\hline$* 1$ & 9 & 22.5 \\
\hline$* 2$ & 19 & 47.5 \\
\hline *3 & 12 & 30 \\
\hline$* 4$ & 0 & 0 \\
\hline
\end{tabular}

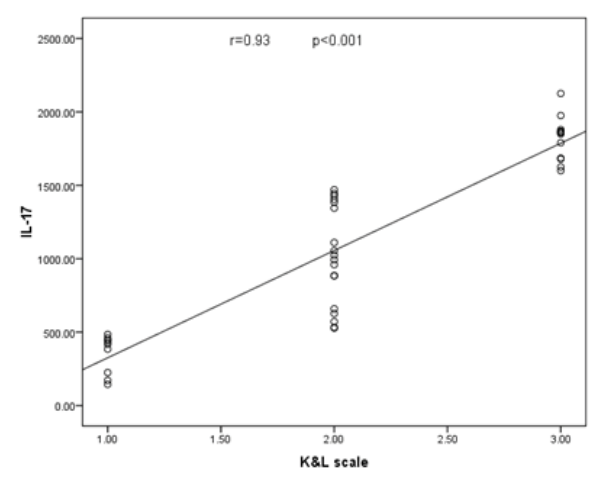

Figure 1. Correlation between serum level of IL-17 and Radiological K\&L scale in patients.

Chen et al. suggested that IL-17 may be among the critical mediators of the disturbed processes implicated in osteoarthritis pathophysiology and stated that in both rheumatoid arthritis and OA, IL-17 can promote cartilage and bone destruction by acting as potent inducer of matrix metalloproteinase and receptor activator of nuclear factor $\mathrm{kB}$ ligand expression in synoviocytes and osteoblasts. In addition, IL17 may be important in recruiting monocytes into the joints of patients, and in promoting the secretion of inflammatory cytokines and chemokines from arthritis synovial tissue. They also suggested that IL-17 can play a key role in the synergistic effects of TNF- $\alpha$ and IL-1 in those patients [17].

On the other hand, Wang et al. [19] found no difference in serum IL-17 levels in the nonKOA patients. At the same time, he found marked increased IL-17 values in the synovium of patients with $\mathrm{KOA}$ in comparison to those in non-KOA cases. They stated that IL-17 may have roles to play in knee OA. And up to 20 


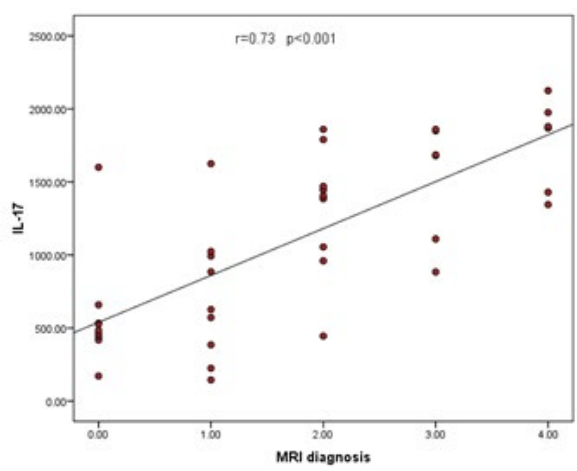

Figure 2. Correlation between serum level of IL-17 and Cartilage degradation using MRI.

$\%$ of chondrocytes in cartilage samples from patients with OA expressed IL-17R. An in vitro study done by Honorati et al. also suggested that IL-17 induced the release of chemokines by chondrocytes and synovial fibroblasts, contributing to cartilage breakdown and synovial infiltration in OA [19].

To assess the variable range of disease severity in cases with osteoarthritis, we studied in details the relation between interleukin-17 and disease function using WOMAC scale. The results of this study revealed significant relation between interleukin 17 and WOMAC scale, this was in agreement with $[20,21]$. The relation between interleukin-17 and disease severity in our cases using Lequesne et al. scale also revealed a highly significant relation between them and this was in agreement with $[19,22,23]$.

Despite the agreement of several studies with the present results, Chen et al. [17] found that the level of IL-17 that is present in synovium only was severely correlated with Lequesne index in his research. This may be caused by the elevated local release of IL-17 by chondrocytes and synovium in joints and extra articular tissue [17]. OA is among the most common reasons of pain and physical disability in elderly. Interestingly, IL-17 has also been recognized as a pain sensitizer in arthritic mice replica [18].

Therefore we studied the relation between interleukin-17 and pain in primary osteoarthritis using VAS pain scale, our results revealed positive correlation between pain and the concentrations of interleukin-17 which means that the patients with higher level of interleukin-17 complained of more painful knee joints. This agreed with Kim and Moalem-Taylor [3] who found that peripheral injection of IL-17 enhances sensitivity of pain in immature animals, and also activates the function of immune cells. They showed also that IL-17 mediate articular mechanical hyper nociception in a replica of Ag-induced joint inflammation. Miller and Malfait [24] also suggested that IL-17 may have a significant act in mechanical pain.

Mathiessen and Conaghan [25] also suggested that inflammation increases the response of peripheral nociceptive neurons, resulting in elevated pain sensitivity and therefore leads to heightened pain experience. On the contrary, Torres et al. [26] stated that pain may not be directly related to IL-17 itself as it may be related to other factors occurring in pathology of osteoarthritis such as bone attrition. Moreover, Link et al. [27] also suggested that it is possibly related to persons handling rather than knee particular pain measure.

Since osteoarthritis is one of the most common causes of disability. Evaluation of cartilage destruction is crucial for observing the disease progression and evaluating the treatment routes in osteoarthritis [28]. According to previous studies, IL-17 increases the release of inflammatory cytokines and adipocytokines, promotes the release of Matrix Metallo Proteinase (MMP), inhibits chondrocyte proliferation, and stimulates Nitric Oxide (NO) and osteoclastogenic cytokine production, such as IL-1 $\beta$ and IL6. Additionally, it stimulates osteoclast and inhibits osteoblast activity as a result it enhances radiographic progression and cartilage degradation [29].

The present study revealed strong link between the concentrations of IL-17 and Kellgren and Lawrence scale in cases with primary knee OA. This agrees with other studies [19,22]. Similarly, a study done by Cooper et al. [30] showed that the existence of interleukin 17 at baseline was highly correlated to the baseline KL X-ray grade. Furthermore, Mathiessen and Conaghan [25] showed that IL-17 was noticeable in serum samples from knee OA patients, and found a positive link between IL-17 levels and the severity of knee osteoarthritis using KL grade.

Chen et al. [17] found that when cases were arranged according to radiographic Kellgren and Lawrence grade, IL-17 serum levels were considerably elevated in cases with grade $4 \mathrm{OA}$ than in healthy volunteers $(\mathrm{P}<0.05)$. However, they found no notable differences among cases with grade 2 or grade $3 \mathrm{OA}$ and healthy volunteers. Several proofs suggest that synovial inflammation and the resultant proinflammatory effectors such as IL-17 are crucial in the 
pathophysiology of osteoarthritis with effects on articular cartilages. Recent imaging devices such as MRI have proved high popularity of 'macroscopic' inflammatory cells and have supported the role of synovial inflammation as an active factor of the osteoarthritic disease, together with both structural progression and pain [25].

MRI gives the chance to observe the cartilage, ligaments, menisci, synovial effusion, and bone marrow lesions closely. Furthermore, MRI is better than $\mathrm{x}$-ray at showing bone cysts, osteophytes, and attrition [28]. Our study revealed that the values of IL-17 were higher in cases with increased degree of cartilage degradation as shown using MRI. Similarly, Lubbeke et al. [31] in their study proved that IL-17 affects cartilage matrix turnover, induces angiogenesis in articular tissue, and also amplifies joint inflammation. Furthermore, a study done by Wang et al. [19] on symptomatic knee OA patients revealed that serum IL-17 was highly related to cartilage destruction at almost all sites (except for medial tibial) after adjusting of age, sex, and BMI. They suggested that metabolic and inflammatory factors may play roles in cartilage loss and bone abnormalities in patients with knee OA [19].

Therefore, it's obvious that IL-17 affects the chondrocytes of OA joints at many levels, since it encourages the release of proinflammatory chemokines like IL-1 $\beta$ and TNF- $\alpha$ by chondrocytes and synovial fibroblasts, leading to cartilages destruction and synovium infiltration in OA. In addition, IL-17 and TNF- $\alpha$ also appear to activate the chondrocytes in a similar manner and the former could be a reason to initiate the changes at cartilage level resulting in cartilage destruction in osteoarthritis [32].

Finally, according to the results of the present study and the studies previously mentioned, there is a strong relation between serum level of IL-17 and primary knee osteoarthritis and this may reflect the degree of severity of the disease to some extent.

\section{Recommendations}

Further investigations are needed to assess the role of IL-17 antagonists in ameliorating disease progression.

\section{References}

1. Kapoor M, Martel-Pelletier J, Lajeunesse D et al. Role of proinflammatory cytokines in the pathophysiology of osteoarthritis. Nat. Rev. Rheumatol. 7(1), 33-42 (2011).
2. Salehi-Abari I. ACR revised criteria for early diagnosis of knee osteoarthritis (2016).

3. Kim CF, Moalem-Taylor G. Interleukin-17 contributes to neuroinflammation and neuropathic pain following peripheral nerve injury in mice. J. Pain. 12(3), 370-383 (2011).

4. Osta B, Benedetti G, Miossec P. Classical and paradoxical effects of TNF- $\alpha$ on bone homeostasis. Front. Immunol. 5, 48 (2014).

5. $\mathrm{Qu} \mathrm{N}, \mathrm{Xu}$ M, Mizoguchi I et al. Pivotal roles of T-helper 17-related cytokines, IL-17, IL-22, and IL-23, in inflammatory diseases. Clin. Dev. Immunol. 2013, 1-13 (2013).

6. Pulsatelli L, Addimanda $\mathrm{O}$, Brusi $\mathrm{V}$ et al. New findings in osteoarthritis pathogenesis: Therapeutic implications. Ther. Adv. Chronic. Dis. 4(1), 23-43 (2013).

7. Zhang W, Doherty M, Peat G et al. EULAR evidence-based recommendations for the diagnosis of knee osteoarthritis. Ann. Rheum. Dis. 69(3), 483-489 (2010).

8. Gunn AA. Antibiotics in biliary surgery. Br. J. Surg. 63(8), 627-630 (1976).

9. Jones AC, Doherty M. The treatment of osteoarthritis. Br. J. Clin. Pharmacol. 33(4), 357363 (1992).

10. Abhishek A, Doherty M. Diagnosis and clinical presentation of osteoarthritis. Rheum. Dis. Clin. North. Am. 39(1), 45-66 (2013).

11. Hawker GA, Mian S, Kendzerska $\mathrm{T}$ et al. Measures of adult pain: Visual analog scale for pain (VAS Pain), numeric rating scale for pain (nrs pain), mcgill pain questionnaire (mpq), short-form mcgill pain questionnaire (sf-mpq), chronic pain grade scale (cpgs), short form36 bodily pain scale (SF). Arthritis. Care. Res. 63(S11), S240-S252 (2011).

12. Theiler R, Spielberger J, Bischoff HA et al. Clinical evaluation of the woman 3.0 OA index in numeric rating scale for meet using computerized touch screen version. $\bigotimes$ Osteoarthr. Cartil. 10(6), 479-481 (2002).

13. Leqense MG, Mery C, Samson M et al. Indexes of severity for osteoarthritis of the hip and knee: Validation-value in comparison with other assessment tests. Scand. J. Rheumatol. 16(suppl 65), 85-89 (1987).

14. Kellgren JH, Lawrence JS. Radiological assessment of osteo-arthrosis. Ann. Rheum. Dis. 16(4), 494-502 (1957).

15. Peterfy CG, Guermazi A, Zaim S et al. Wholeorgan magnetic resonance imging score (WOMS) of the knee in osteoarthritis. Osteoarthr. Cartil. 12(3), 177-190 (2004). 
16. Westergren A (1957) Diagnostic tests: The erythrocyte sedimentation rate range and limitations of the technique. Triangle. 3(1), 2025 (1957).

17. Chen B, Deng Y, Tan Y et al. Association between severity of knee osteoarthritis and serum and synovial fluid interleukin 17 concentrations. $J$. Int. Med. Res. 42(1), 138-144 (2014).

18. Snelling SJB, Bas S, Puskas GJ et al. Presence of IL-17 in synovial fluid identifies a potential inflammatory osteoarthritic phenotype. PloS one. 12(4), e0175109 (2017).

19. Wang K, Xu. J, Cai JJ et al. Serum levels of interleukin-17 and adiponectin are associated with infrapatellar fat pad volume and signal intensity alteration in patients with knee osteoarthritis. Arthritis. Res. Ther. 18(1), 193 (2016).

20. Askari A, Naghizadeh MM, Homayounfar R et al. Increased serum levels of IL-17A and IL-23 are associated with decreased vitamin D3 and increased pain in osteoarthritis. PloS one. 11(11), e0164757 (2016).

21. Imamura M, Ezquerro F, Alfieri FM et al. Serum levels of proinflammatory cytokines in painful knee osteoarthritis and sensitization. Int. J. Inflam. 2015, 1-8 (2015).

22. Malemud CJ. The biological basis of osteoarthritis: State of the evidence. Curr. Opin. Rheumatol. 27(3), 289-294 (2015).

23. Ceccarelli F, Perricone C, Pacucci VA et al. AB0036 Association between synovitis and inflammatory citokines serum levels in a cohort of patients affected by primary knee osteoarthritis (2017).

24. Miller RE, Miller RJ, Malfait AM. Osteoarthritis joint pain: the cytokine connection. Cytokine. 70(2), 185-193 (2014).

25. Mathiessen A, Conaghan PG. Synovitis in osteoarthritis: Current understanding with therapeutic implications. Arthritis. Res. Ther. 19(1), 18 (2017).

26. Torres L, Dunlop DD, Peterfy C et al. The relationship between specific tissue lesions and pain severity in persons with knee osteoarthritis. Osteoarthr. Cartil. 14(10), 1033-1040 (2006).

27. Link TM, Steinbach LS, Ghosh $S$ et al. Osteoarthritis: MR imaging findings in different stages of disease and correlation with clinical findings. Radiology. Radiological Society of North America. 226(2), 373-381 (2003).

28. Raynauld JP, Martel-Pelletier J, Berthiaume MJ et al. Quantitative magnetic resonance imaging evaluation of knee osteoarthritis progression over two years and correlation with clinical symptoms and radiologic changes. Arthritis. Rheum. 50(2), 476-487 (2004).

29. Liu Y, Peng H, Meng Z et al. Correlation of IL-17 level in synovium and severity of knee osteoarthritis', Medical science monitor. Int. Med. J. Exp. Clin. Res. 21, 1732-1736 (2015).

30. Cooper C, Snow S, McAlindon TE et al. Risk factors for the incidence and progression of radiographic knee osteoarthritis. Arthritis. Rheum. 43(5), 995-1000 (2000).

31. Lubbeke A, Bas S, Puskas GJ et al. Presence of interleukin-17 in osteoarthritis: Does it indicate a different osteoarthritis phenotype. Osteoarthr. Cartil. 23, A321 (2015).

32. Chang SH, Dong C. Signaling of interleukin-17 family cytokines in immunity and inflammation. Cell. Signal. 23(7), 1069-1075 (2011). 